\title{
O papel do farmacêutico na prevenção de erros de medicação
}

\author{
The pharmacist's role in preventing medication errors \\ El papel del farmacéutico en la prevención de errores de medicación
}

Fabiana Sari Ferreira

ORCID: https://orcid.org/0000-0002-2358-6919 Universidade Estadual do Oeste do Paraná, Brasil

E-mail: sari.fabiana@gmail.com

Thais Arnoni Pereira

ORCID: https://orcid.org/0000-0003-4422-8915 Universidade Estadual de Maringá, Brasil E-mail: thaisarnoni07@gmail.com

Beatriz Paludo de Souza ORCID: https://orcid.org/0000-0002-0683-0596 Universidade Estadual do Oeste do Paraná, Brasil E-mail: bia.paludo@hotmail.com

Andréia Cristina Conegero Sanches ORCID: https://orcid.org/0000-0002-3108-7300 Hospital Universitário do Oeste do Paraná, Brasil E-mail: andreiaconegero@gmail.com

\begin{abstract}
Resumo
Visando a promoção de práticas seguras e a geração de indicadores para o monitoramento e avaliação do processo da utilização dos medicamentos, o objetivo deste trabalho foi identificar os erros de prescrição e dispensação evitados pela equipe farmacêutica a fim de contribuir para a qualidade do serviço. Trata-se de uma análise transversal realizada na farmácia central em um hospital escola no período de 01 janeiro a 31 dezembro de 2019. Foram avaliados os registros de intervenções farmacêuticas e da conferência das medicações antes da dispensação. Os dados coletados produziram indicadores que foram analisados através do Diagrama de Pareto e confrontados com os eventos notificados na instituição. Os erros de prescrição mais detectados pela intervenção farmacêutica foram a frequência e a dose inadequada. Os erros prevenidos durante a conferência dos pacotes de medicações foram a falta de medicamento prescrito e troca de horário. Além disso, das notificações relacionadas aos erros de medicação, foram observados que $32,88 \%$ eram referentes aos erros de prescrição, $41,10 \%$ aos erros de dispensação e $26,03 \%$ aos erros de administração. Mesmo com a subnotificação, foram registrados 3343 eventos prevenidos pela equipe farmacêutica, mostrando que sua atuação é indispensável e faz parte das estratégias de prevenção de eventos adversos no âmbito hospitalar.
\end{abstract}

Palavras-chave: Evento adverso; Near miss; Segurança do paciente.

\begin{abstract}
Aiming at promoting safe practices and generating indicators for monitoring and evaluating the process of drug use, the objective of this work was to identify the prescription and dispensing errors avoided by the pharmaceutical team in order to contribute to the quality of the service. This is a cross-sectional analysis carried out at the central pharmacy in a teaching hospital from January 1st to December 31th, 2019. The records of pharmaceutical operations and the checking of medications before dispensing were obtained. The collected data produced indicators that were analyzed using the Pareto Diagram and compared with the events notified at the institution. The most frequent prescription errors avoided by pharmaceutical intervention was the frequency and the inadequate dose. The errors prevented during the checking of the medication packages were the lack of a prescribed medication and a change of schedule. In addition, from notifications related to medication errors, it was observed that $32.88 \%$ were related to prescription errors, $41.10 \%$ to dispensing errors and $26.03 \%$ to administration errors. Even with underreporting, 3343 events prevented by the pharmaceutical team were recorded, showing that their performance is essential and is part of the strategies for preventing adverse events in the hospital environment.
\end{abstract}

Keywords: Adverse reactions; Near miss healthcare; Patient Safety.

\section{Resumen}

Con el objetivo de promover prácticas seguras y generar indicadores para el seguimiento y evaluación del proceso de uso de medicamentos, el objetivo de este trabajo fue identificar los errores de prescripción y dispensación evitados por el equipo farmacéutico para contribuir a la calidad del servicio. Se trata de un análisis transversal realizado en la 
farmacia central de un hospital universitario del 1 de enero al 31 de diciembre de 2019. Se obtuvieron registros de operaciones farmacéuticas y control de medicamentos antes de su dispensación. Los datos recolectados produjeron indicadores que fueron analizados mediante el Diagrama de Pareto y comparados con los eventos notificados en la institución. Los errores de prescripción más frecuentes evitados por la intervención farmacéutica fue la frecuencia y la dosis inadecuada. Los errores que se evitaron durante la revisión de los paquetes de medicamentos fueron la falta de medicación prescrita y el cambio de horario. Además, de las notificaciones relacionadas con errores de medicación, se observó que el $32,88 \%$ estaban relacionados con errores de prescripción, el $41,10 \%$ con errores de dispensación y el $26,03 \%$ con errores de administración. Incluso con el subregistro, se registraron 3.343 eventos prevenidos por el equipo farmacéutico, lo que demuestra que su desempeño es fundamental y forma parte de las estrategias de prevención de eventos adversos en el entorno hospitalario.

Palabras clave: Evento adverso; Near miss salud; Seguridad del paciente.

\section{Introdução}

Visando a promoção de práticas seguras, o Programa Nacional de Segurança do Paciente (MS, 2013), propõe a geração de indicadores para o monitoramento e avaliação das atividades desempenhadas de todas as etapas do processo da utilização dos medicamentos (ISMP, 2016), e diante da possibilidade de prevenção dos erros de medicação, o Protocolo de Segurança na Prescrição, Uso e Administração de Medicamentos tem a finalidade de auxiliar na promoção destas práticas nos estabelecimentos de saúde (MS \& ANVISA, 2013).

Como a atuação farmacêutica hospitalar vai além da gestão dos medicamentos e da otimização da farmacoterapia, a qualidade e segurança da assistência permitem que o farmacêutico também contribua na redução dos erros de prescrições, de dispensação e administração de medicamentos e ainda promove sua participação nas equipes multiprofissionais (Fernandes, 2019).

Nesse contexto, mesmo quando se avalia um sistema amplamente complexo, as barreiras podem ser ultrapassadas por apresentar pontos fracos não intencionais. Uma série de eventos incidentes interligados, pode ocasionar dano ao paciente (Perneger, 2005; Reason, 2000). Assim, os erros de medicação podem provocar importantes agravos à saúde do paciente, também podem ser eventos evitáveis quando identificados antes de atingir o paciente, neste caso são definidos como Near Miss, que poderiam ter prejudicado o paciente, mas o evento adverso foi evitado de alguma forma (Anacleto, Rosa, Neiva, \& Martins, 2017; Benson \& Grieve, 2021).

Desta forma, este estudo se justifica pela necessidade de avaliação da assistência farmacêutica por meio dos indicadores que oportunizam a implementação de estratégias para a correção de falhas e aperfeiçoamento do serviço como orienta o ISMP (2016). E com isso, o objetivo do presente trabalho foi identificar os erros de prescrição e dispensação evitados pela equipe farmacêutica para contribuir no reparo dos processos e comparar os resultados encontrados com o que foi notificados ao Núcleo da Gestão da Qualidade e Segurança do Paciente (NGQ/NSP), garantindo a melhor assistência ao paciente.

\section{Metodologia}

Trata-se de uma análise documental, transversal e retrospectiva de caráter quantitativo (Pereira, Shitsuka, Parreira, \& Shitsuka, 2018), realizada na farmácia central do Hospital Universitário do Oeste do Paraná (HUOP) de nível terciário pertencente à Universidade Estadual do Oeste do Paraná (UNIOESTE), localizado no município de Cascavel. O hospital possui em torno de 235 leitos atendidos em sua totalidade pelo Sistema Único de Saúde (SUS) e o serviço de farmácia hospitalar é composto pela Central de Abastecimento Farmacêutico, Farmácia Central e Farmácias Satélites.

As Farmácias Satélites, durante o desenvolvimento desta pesquisa, eram apenas duas. Uma referente ao Centro Cirúrgico e Obstétrico e outra ao Pronto Socorro. No entanto, no ano de 2020, após o desenvolvimento desta pesquisa, foi inaugurada a Farmácia Satélite das Unidades de Terapia Intensiva (UTI). Além disso, os Serviços de Farmácia Clínica e 
Farmacovigilância que complementam a assistência farmacêutica neste hospital.

O serviço de farmácia registrou diariamente as intervenções farmacêuticas realizadas durante a validação das prescrições médicas realizadas no período de 01 de janeiro de 2019 a 31 de dezembro de 2019. Os dados foram tabulados em planilha Microsoft Excel® e produzidos indicadores de erros prescrições, conforme Figura 1.

Figura 1. Fluxograma do processo de validação farmacêutica das prescrições eletrônicas.

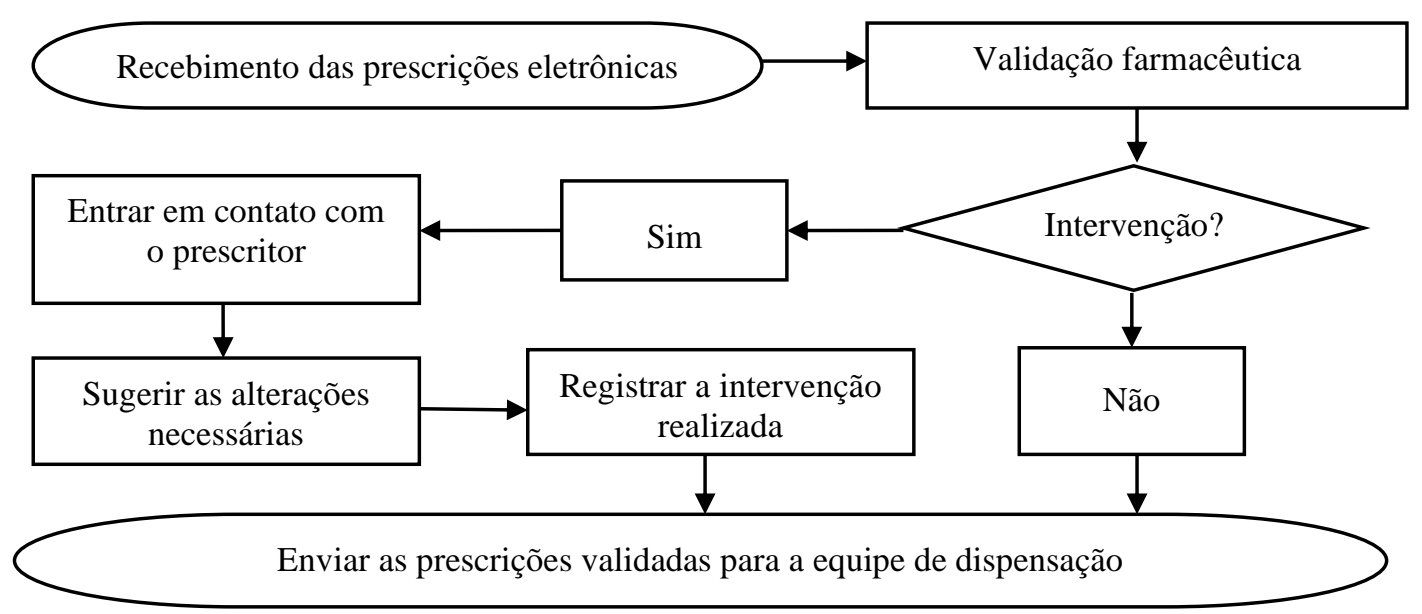

Fonte: Autores.

Como o sistema de distribuição de medicamentos do hospital é individualizado, após validação da prescrição, os medicamentos eram separados e a prescrição eletrônica atendida individualmente através do código de barras dos medicamentos com o auxílio do sistema eletrônico de gestão hospitalar (Sistema Philips Tasy®, Blumenau/SC, versão 3,01,1731 09/10/2018) para baixa deles no estoque da farmácia e cobrança da conta do paciente, que no caso deste hospital, é por meio do SUS.

Na sequência, os funcionários confeccionavam pacotes com as medicações separadas por turno (manhã, tarde e noite) para cada paciente, após esta etapa, estes eram conferidos pelos farmacêuticos. Após a conferência de todos os pacotes de medicamentos, estes são enviados para as unidades de internação. Cada erro encontrado na etapa de conferência foi tabulado em planilha Microsoft Excel@ e produzidos indicadores de erros na confecção dos pacotes de medicação. Todo esse processo no fluxo (Figura 2) da farmácia hospitalar tem a finalidade de prevenir erros de dispensação, conforme a RDC 36/2013 do Ministério da Saúde.

Figura 2. Fluxograma do processo de dispensação dos medicamentos prescritos.

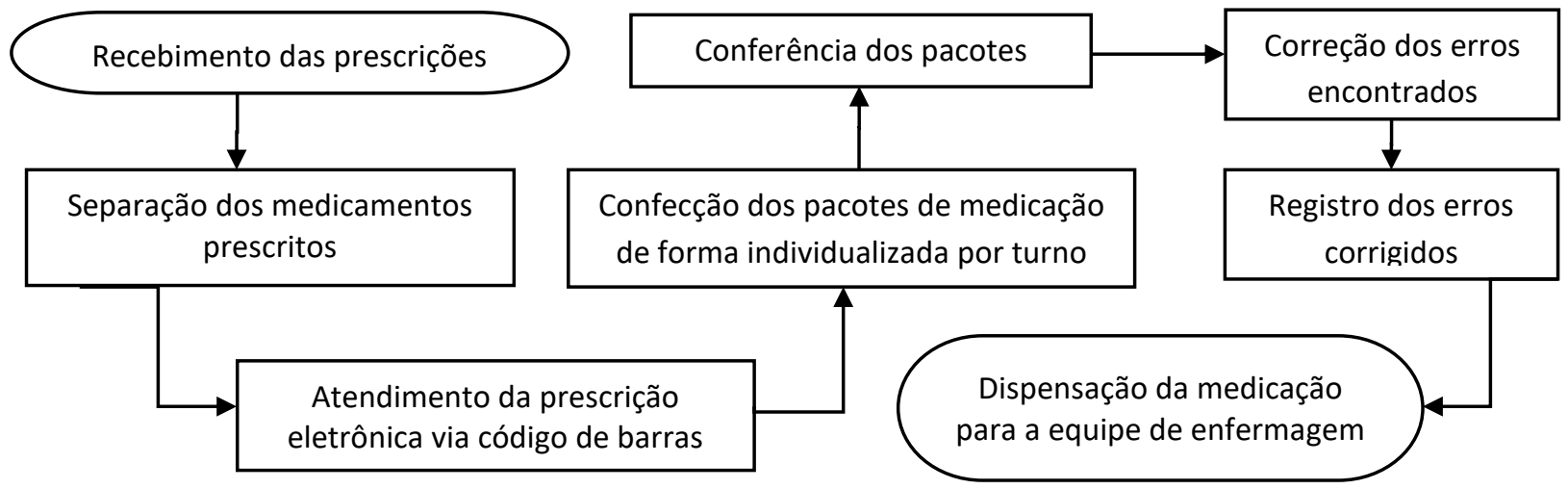

Fonte: Autores. 
A análise estatística utilizada com o intuito de direcionar e priorizar as ações para melhoria dos processos, foi o Diagrama de Pareto (Haino, 2013) realizado com auxílio do software Microsoft Excel®. Além disso, como é dever do Núcleo de Segurança do Paciente (NSP) notificar os eventos adversos decorrentes da prestação do serviço de saúde (MS, 2013), os dados encontrados foram confrontados com os eventos notificados no mesmo período ao NGQ/NSP do hospital e classificados como erro de prescrição ou dispensação. Este estudo faz parte do projeto aprovado pelo Comitê de Ética em Pesquisa institucional, sob parecer $\mathrm{n}^{\circ} 1.872 .685$ de 16/12/2016.

\section{Resultados e Discussão}

Considerando o quantitativo documentado durante a conferência dos medicamentos pelo farmacêutico, foram registradas 43.319 prescrições médicas e 836 intervenções farmacêuticas, correspondendo a aproximadamente 01 intervenção para cada 51 prescrições, sendo que a $57,54 \%$ das vezes $(n=481)$ foi feita a solicitação da possibilidade de substituição por outro medicamento ou forma farmacêutica devido à falta de algum medicamento. Pesquisas em hospitais públicos, como a de Dias e colaboradores (2019) e Araujo et al (2017) mostraram respectivamente, a necessidade de substituição motivada por falta do fármaco em apenas $2 \%$ e 8,99\% dos casos, no entanto, foram avaliadas apenas as intervenções farmacêuticas em Unidades de Terapia Intensiva (UTI), diferentemente do que ocorreu no presente trabalho, em que os dados produzidos foram referentes à todas as unidades de internação do hospital.

Decorrente do desabastecimento ou por conta de baixa disponibilidade de financiamento ou problemas de gestão local da assistência, a falta do produto para ser dispensado representa um problema importante, do direito à saúde (Chaves, Chaves, Vianna, \& Oliveira, 2019). Alguns autores relatam dificuldades logística devido a restrição na implantação de novas tecnologias e obtenção de adequada infraestrutura por se tratar de organização pública, tanto na escassez de recursos financeiros e humanos, quanto a cultura do funcionalismo (de Fátima Silva, Afonso, Veloso e Sousa, \& Pellizzaro Dias Afonso, 2018).

Apesar disso, é possível implantar medidas de melhorias através da conscientização dos envolvidos, garantindo enfoque interdisciplinar, revisando os processos logísticos e as estratégias com potencial minimização dos eventos de ruptura de estoques (Cação, Bokehi, Futuro, \& De Castilho, 2020; Lima \& Câmara, 2016).

Durante o período avaliado, observamos que 51,76\% das intervenções farmacêuticas solicitando substituição devido à falta de algum medicamento eram referentes ao omeprazol $(\mathrm{n}=125)$ e a ranitidina $(\mathrm{n}=124)$, mas que não tiveram seus motivos registrados e que, para identifica-los implicaria em um estudo mais específico junto da Central de Abastecimento Farmacêutico (CAF), mas se sabe que a Agência Nacional de Vigilância Sanitária (ANVISA) suspendeu em 2019 a importação do insumo farmacêutico ranitidina e consequentemente impactou no desabastecimento para ambas as formas farmacêuticas padronizadas no hospital (comprimido e injetável) e, consequentemente a instituição sofreu com a falta, tanto de ranitidina que não tinha possibilidade de compra, quanto do omeprazol que teve seu consumo aumentado pela falta da ranitidina (ANVISA, 2019).

Independente disso, Araújo e colaboradores (2020) mostraram que as intervenções farmacêuticas podem contribuir significativamente para o uso racional de omeprazol intravenoso, que pode servir de modelo para ser implantado na instituição, tanto para o omeprazol, quanto para outros medicamentos em questão, de modo a colaborar com as estratégias de logística da CAF.

No entanto, se considerarmos um recorte das intervenções realizadas $(n=327)$, onde se excluem as solicitações de troca por falta/desabastecimento, é possível identificar que a intervenção farmacêutica mais realizada foi devido a prescrição de medicamento com frequência inadequada $(17,43 \% ; \mathrm{n}=57)$, seguido por medicamento com dose inadequada $(16,21 \% ; \mathrm{n}=$ 
53), medicamentos com via de administração inadequada para o paciente $(11,62 \% ; \mathrm{n}=38)$, para confirmação da dose prescrita $(10,09 \% ; n=33)$, solicitação de suspensão do medicamento $(10,09 \% ; n=33)$ e outros menos frequentes conforme observa-se na Tabela 1.

Tabela 1. Classificação dos erros de prescrição registrados durante a validação farmacêutica em hospital de ensino no ano de 2019.

\begin{tabular}{lcccccccc}
\hline & CM & MAT & PED & OC & NV & UTI & TOTAL & $\%$ \\
\hline Medicamento não indicado & 0 & 2 & 0 & 1 & 2 & 1 & 6 & 1,83 \\
Duplicidade terapêutica & 4 & 3 & 2 & 7 & 2 & 2 & 20 & 6,12 \\
Forma farmacêutica inadequada & 4 & 0 & 0 & 1 & 1 & 2 & 8 & 2,45 \\
Horário inadequado & 9 & 3 & 1 & 7 & 4 & 0 & 24 & 7,34 \\
Frequência inadequada & 17 & 7 & 2 & 19 & 11 & 1 & 57 & 17,43 \\
Via de administração inadequada & 10 & 1 & 3 & 17 & 5 & 2 & 38 & 11,62 \\
Dose inadequada & 19 & 8 & 6 & 6 & 11 & 3 & 53 & 16,21 \\
Medicamento não padrão & 0 & 6 & 3 & 6 & 8 & 5 & 28 & 8,56 \\
Suspensão do medicamento & 5 & 2 & 0 & 10 & 14 & 2 & 33 & 10,09 \\
Confirmação da dose & 8 & 3 & 3 & 7 & 5 & 7 & 33 & 10,09 \\
Medicamento com o paciente & 4 & 5 & 4 & 7 & 1 & 1 & 22 & 6,73 \\
Não classificada & 1 & 1 & 0 & 1 & 0 & 2 & 5 & 1,53 \\
TOTAL & 81 & 41 & 24 & 89 & 64 & 28 & 327 & \\
Taxa de erro por unidade (\%) & 24,77 & 12,54 & 7,34 & 27,22 & 19,57 & 8,56 & & \\
\hline
\end{tabular}

Legenda: CM - Clínica Médica; MAT - Maternidade; PED - Pediatria; OC - Ortopedia e Cirúrgica; NV - Neurologia e Vascular; UTI Unidade de Terapia Intensiva.

Fonte: Dados da pesquisa.

Apesar da literatura mostrar resultados semelhantes para intervenção farmacêutica devido a dose inadequada, a exemplo de 17,1\% descrito por Botelho \& Rose (2017) e de 13\% por Dias e Colaboradores (2019), os demais resultados foram bastante dispersos entre os estudos encontrados. Miranda et al (2012) foi o estudo que mais se aproximou no quantitativo de intervenções relacionadas a via de administração, com 8,48\%. Já Maioli \& Santos (2018), publicaram índices maiores dos que verificamos no presente estudo, com 28,2\% das intervenções de posologia, 33,2\% devido a inconsistência da dose e 14,9\% para via de administração.

As intervenções relacionadas a via de administração dos medicamentos têm grande valia e são aliadas à gestão dos recursos junto à CAF. A Switch Therapy, denominação utilizada para a substituição de um medicamento de administração por via intravenosa para via oral, de mesma classe e nível de potência, favorecem a redução significativa nos custos da terapia (Araújo \& Melo, 2018)

Ainda, foi possível observar que, o omeprazol $(n=8)$ foi o medicamento com mais correção de frequência, provavelmente devido a falta do mesmo durante algum período e o maior foco dos farmacêuticos para o ajuste da dose daqueles que estavam fazendo uso inadequado. Já a heparina $(n=22)$ foi o medicamento que teve a via de administração mais prescrita de forma incorreta, pois na instituição é padronizada tanto a apresentação endovenosa como a subcutânea e que poderia provocar danos graves ao paciente se não fosse evitado. Quanto as doses, foi observado frequentemente um despropósito nos medicamentos multidoses como a simeticona $(n=5)$ e a lactulose $(n=5)$, devido a inabilidade digital dos 
profissionais médicos com a prescrição eletrônica, pois na maioria das vezes estava prescrito "frascos" ao invés de "mL" ou "mL" ao invés de "gotas" do medicamento, caracterizando sobredose.

Algumas terapias de antimicrobianos foram identificadas como inadequadas pelos farmacêuticos que realizavam a validação das prescrições e tiveram suas sugestões aceitas pela equipe médica, representando 83,33\% ( $\mathrm{n}=5$ ) daqueles medicamentos que foram classificados como não indicados ao tratamento do paciente. $\mathrm{O}$ tramadol comprimido representou 46,67\% ( $\mathrm{n}=7)$ da prescrição medicamento não padronizado e, a Piperacilina + Tazobactam $(33 \% ; n=3)$ e a Cefazolina (33\%; $\mathrm{n}=3$ ) dos medicamentos que foram suspensos, devido a atenção da equipe na monitorização da antibioticoterapia, suspendendo a prescrição dele quando completado o tempo de tratamento total. A duplicidade terapêutica representada pela ranitidina e omeprazol foi equivalente a $46,15 \%(n=6)$ e, as demais intervenções não foram retratadas por algum medicamento em específico de forma relevante.

Com a finalidade de demonstrar a importância de todas as possibilidades causadoras de um problema, viabilizando um ponto de partida para sua identificação, solução e monitoramento, foi estruturado um Diagrama de Pareto (Haino, 2013), como mostra a Figura 3, a especialidades que mais careceu de Intervenção Farmacêutica para ajuste dos erros de prescrição é a Ortopedia e Cirúrgica, seguida pela Clínica Médica e Neurologia e Vascular.

Figura 1. Análise de Pareto quanto à incidência dos erros de prescrição em hospital de ensino no ano de 2019.

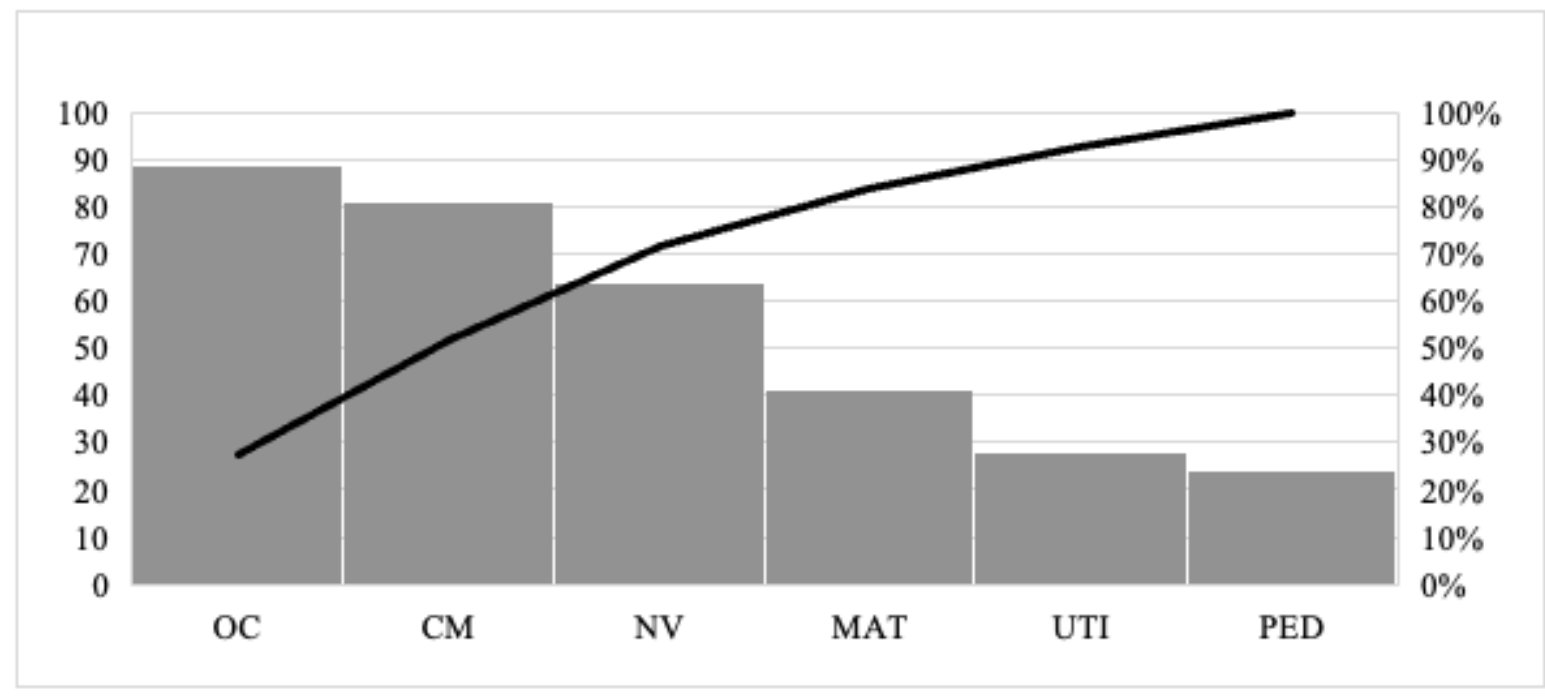

Legenda: OC - Ortopedia e Cirúrgica; CM - Clínica Médica; NV - Neurologia e Vascular; MAT - Maternidade; UTI - Unidade de Terapia Intensiva; PED - Pediatria.

Fonte: Dados da pesquisa.

A prescrição eletrônica contribui para minimização dos erros farmacoterapêuticos prescritos (Rezende et al., 2020) e como a instituição em estudo já implantou esta sistematização, sugerimos como estratégia a educação continuada das equipes, tendo em vista a alta rotatividade de profissionais por questão de o hospital ser uma instituição de ensino e, porque muitas das não conformidades observadas podem ser evitadas com treinamentos no que se refere a utilização do sistema informatizado. Além disso, a participação do farmacêutico hospitalar e clínico nas equipes multiprofissionais é essencial para promover a prevenção e redução de danos aos pacientes, a otimização dos benefícios e recursos relacionados à farmacoterapia e ainda, proporcionar o desenvolvimento da equipe (Vieira, 2019; Ferreira \& Soler, 2020).

Vale ressaltar que, nem sempre as intervenções farmacêuticas resultam numa correção da prescrição pelo médico no primeiro contato, carecendo de uma nova intervenção farmacêutica no dia seguinte para o mesmo erro de prescrição já identificado anteriormente, método o qual também poderia ser otimizado pelo sistema de prescrição eletrônica. 
A partir da validação farmacêutica, os medicamentos foram separados e a prescrição eletrônica atendida individualmente através do código de barras dos medicamentos, para dar sequência à confecção dos pacotes individualizados por paciente e por turno. Ao final, foram conferidos pelos farmacêuticos antes de serem enviados para as unidades de internação um total de 63.082 pacotes de medicações.

Nesta etapa foram identificados em média 5,15\% de erros de dispensação por unidade de internação, ocorrências as quais foram evitadas antes de atingirem o paciente pois foram corrigidos antes de serem dispensados. Conforme descrito na Tabela 2, as unidades de internação que mais apresentaram falhas durante o processo de separação, digitação e confecção dos pacotes de medicação, foram a Unidade de Terapia Intensiva (UTI), seguido pela Neurologia e Vascular e pela Clínica Médica, respectivamente com $7,91 \%, 6,44 \%$ e $5,46 \%$.

Tabela 2. Classificação dos erros de dispensação registrados durante a conferência dos pacotes de medicação pelo farmacêutico em hospital de ensino no ano de 2019.

\begin{tabular}{lcccccccc}
\hline & CM & MAT & PED & OC & NV & UTI & TOTAL \\
\hline $\mathrm{N}^{\circ}$ pacotes conferidos & 13143 & 12141 & 8331 & 13688 & 12073 & 3706 & 63082 \\
Identificação do paciente & 37 & 17 & 10 & 24 & 18 & 11 & 117 & 3,80 \\
Medicamento não prescrito & 42 & 20 & 26 & 39 & 57 & 16 & 200 & 6,50 \\
Falta de medicamento prescrito & 215 & 102 & 92 & 172 & 236 & 132 & 949 & 30,85 \\
Dose incorreta & 96 & 29 & 43 & 72 & 101 & 53 & 394 & 12,81 \\
Troca de horário & 173 & 59 & 63 & 146 & 173 & 52 & 666 \\
Troca da forma farmacêutica & 21 & 6 & 4 & 11 & 33 & 6 & 81,65 \\
Troca de cuba & 86 & 68 & 29 & 105 & 114 & 19 & 421 & 13,69 \\
Pacote repetido & 47 & 59 & 20 & 73 & 45 & 4 & 248 \\
TOTAL & 717 & 360 & 287 & 642 & 777 & 293 & 3076 \\
$\%$ & 5,46 & 2,97 & 3,44 & 4,69 & 6,44 & 7,91 & 8,06 \\
\hline
\end{tabular}

Legenda: CM - Clínica Médica; MAT - Maternidade; PED - Pediatria; OC - Ortopedia e Cirúrgica; NV - Neurologia e Vascular; UTI Unidade de Terapia Intensiva.

Fonte: Dados da pesquisa.

O maior número de erros na confecção dos pacotes de medicação da UTI pode ser justificado pelo fato de que os medicamentos são separados no plantão noturno, onde há o menor número de funcionários na farmácia e as visitas médicas acontecem pela manhã, podendo haver alterações na prescrição que precisam ser corrigidas nos pacotes já confeccionados. Os erros relacionados à Neurologia e Vascular podem ser por conta da maior quantidade de itens das prescrições e, da Clínica Médica devido ao atraso na liberação das prescrições pela equipe médica, apressando a rotina da dispensação na farmácia que, também tem o serviço interrompido com frequência pela equipe de enfermagem para solicitação de medicamentos prescritos "Se Necessário", “A Critério Médico” ou ainda, para buscar materiais médico-hospitalares cujas dispensações não haviam sido programados previamente para um determinado horário.

Acerca da classificação dos de erros identificados e prevenidos, foram registrados com maior frequência a falta de medicamento prescrito $(30,85 \% ; n=949)$ e a troca de horário $(21,65 \% ; n=666)$, que juntamente com a troca de ala $(13,69 \%$ n $=421)$ e a dose incorreta $(12,81 \% ; \mathrm{n}=394)$ favorecem a resolubilidade direcionada pela Análise de Pareto, como está representado na Figura 4. 
Figura 2. Análise de Pareto quanto à incidência dos erros de dispensação em hospital de ensino no ano de 2019.

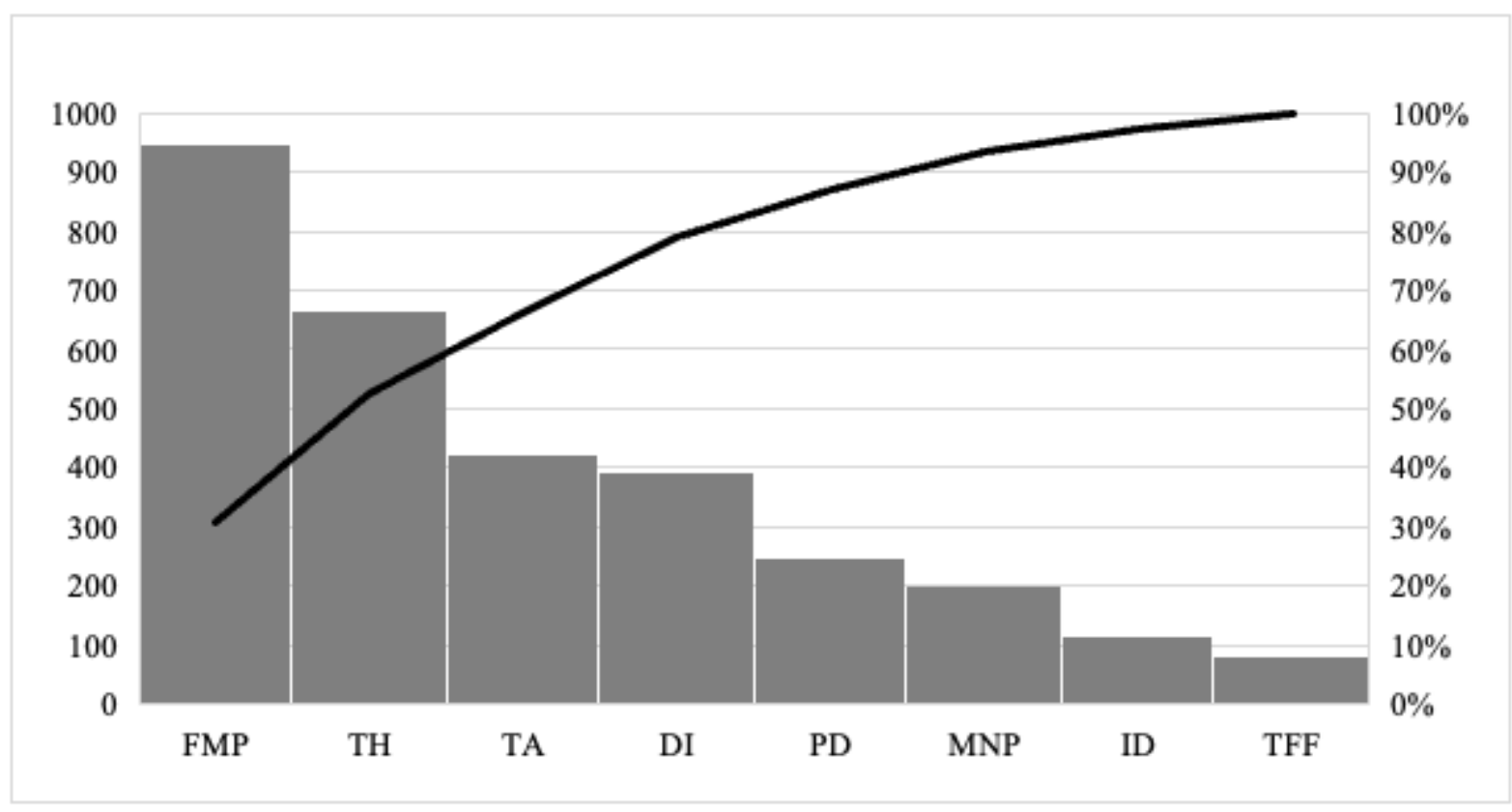

Legenda: FMP - Falta de Medicamento Prescrito; TH - Troca de Horário; TA - Troca de Ala; DI - Dose incorreta; PD - Pacote duplicado; MNP - Medicamento Não Prescrito; ID - Identificação do paciente; TFF - Troca de Forma Farmacêutica.

Fonte: Dados da pesquisa.

Um estudo semelhante publicado por Sirtoli e colaboradores (2019) avaliou os erros de dispensação da farmácia central desta mesma instituição, porém no período de 2015. Tal estudo também mostrou a incidência da falta de medicamento prescrito e troca de horário como os dois fatores majoritários, com 41,66 e 30\%, respectivamente.

A falta de medicamento prescrito, também descrito por "erro de omissão" na literatura, se destaca como principal erro de dispensação relatado em outros estudos (Rissato, 2012; Sirtoli et al., 2019), além disso, em um estudo comparativo, foi observado que na modalidade executada em 2009 que atendia os três turnos, registrou 13,1\% de troca de horário, enquanto que no ano de 2012 foi adotado o lote de dispensação, que anulou esse tipo de erro (Galvão, Oliveira, Carvalho, \& Araújo, 2012).

Apesar das taxas serem menores que os resultados no ano de 2015, muitas são as razões para esses dados se repetirem, como por exemplo a sobrecarga de atividades, a rotatividade dos profissionais e o déficit nos treinamentos. Além disso, como medida preventiva, foram investigadas as possíveis fragilidades na rotina, que proporcionou em meados do ano de 2020 a implantação de uma nova Farmácia Satélite para atender exclusivamente as Unidades de Terapia Intensiva, desafogando a rotina da Farmácia Central e possibilitando a introdução do processo de dupla checagem dos pacotes de medicações no momento de sua dispensação juntamente com a equipe de enfermagem.

Quando se trata de qualidade e segurança do paciente, a notificação de incidentes visam a identificação das causas, para que as ações preventivas e corretivas possam ser discutidas e implementadas, com realização da educação continuada, buscando a melhoria, tanto da assistência, como a efetiva segurança do paciente e do profissional, quanto dos processos organizacionais das instituições de saúde (Prates \& Malta, 2017; Resende et al., 2020).

Contudo, durante o ano de 2019, foram notificados para o NGQ/NSP apenas 73 incidentes relacionados a erros de medicação como ilustrado na Figura 5. 
Figura 3. Quantidade de incidentes relacionados a erro de medicação notificados ao Núcleo da Gestão da Qualidade e Segurança do Paciente em hospital de ensino no ano de 2019.

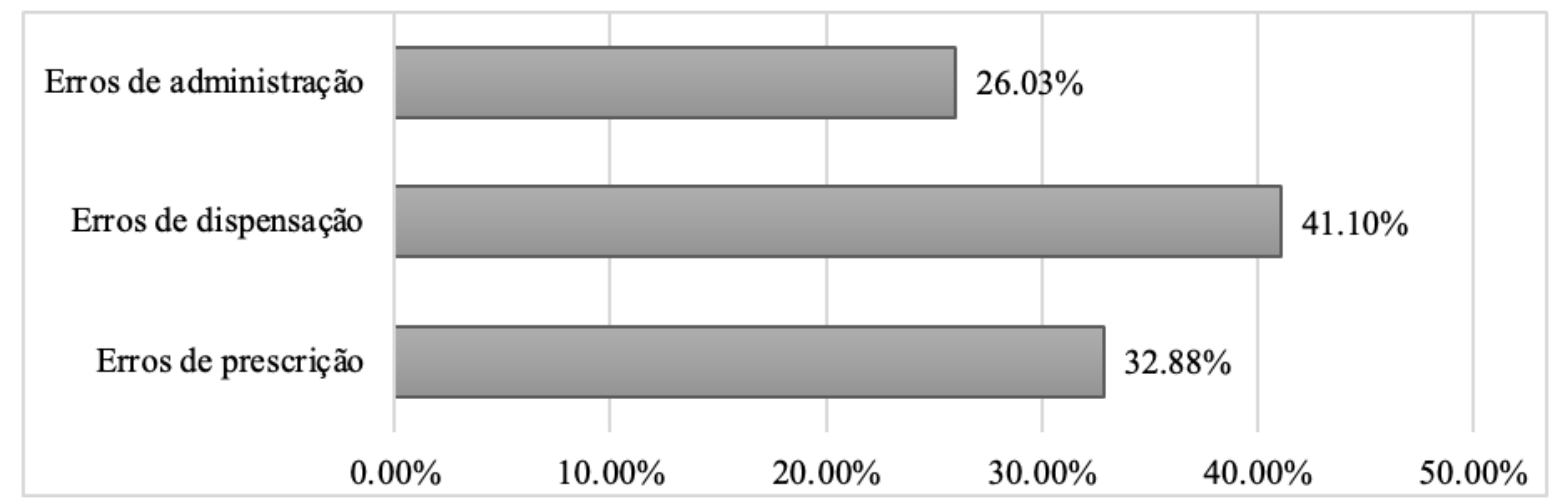

Fonte: Dados da pesquisa.

Os erros de prescrição médica correspondem a 32,88\% $(n=24)$ das notificações referentes a erros de medicação, sendo mais frequentes aquelas notificações relacionadas a falta de prescrição para o paciente $(\mathrm{n}=9)$, seguido do erro ou falta de informações necessárias na prescrição $(n=5)$, prescrição desnecessária de medicamentos $(n=5)$ e outros menos frequentes, mas não menos importantes, como tempo de tratamento, dose incorreta ou medicamento errado/desnecessário. Já, 41,10\% $(\mathrm{n}=$ 30) são referentes aos erros de dispensação, sendo que as notificações referentes ao não envio de medicações a mais incidente $(n=10)$, seguido por dispensação de medicamento trocado/errado $(n=9)$ e atraso na entrega $(n=5)$, além de outras menos frequentes como forma farmacêutica trocada, falta de medicamento, identificação inadequada do medicamento ou horário trocado.

A subnotificação é evidente, visto que foram identificados 267 erros de prescrição durante a validação farmacêutica e foram registrados pelo NGQ/NSP ao todo 24 notificações, das quais, apenas 2 tinham sido realizadas pelo serviço de farmácia hospitalar e, de forma semelhante deve acontecer com os erros de dispensação, que voltam para a farmácia para serem corrigidos e não são notificados, pois as taxas de eventos prevenidos são extremamente maiores que os erros notificados.

Estudos indicam que a subnotificação ainda é sustentada pela vergonha e medo da cultura de segurança frágil e de ações punitivas (Jesus, Morais, \& Almeida, 2020; Mourão \& Oliveira, 2019), além do descaso dos profissionais e da sobrecarga de trabalho (Mascarenhas, Anders, Gelbcke, Lanzoni, \& Ilha, 2019). Esses fatores também refletem a realidade da instituição em questão, no entanto, a cultura punitiva está sendo elucidada através de treinamentos rotineiros com as equipes e da realização de feedback com as partes envolvidas nas notificações.

Possivelmente, os erros de administração não relatados pela enfermagem também estariam subnotificados, pois foram registradas apenas 19 notificações com erros de administração de medicamentos (26,03\%), qualificados em: não administração de medicamentos $(\mathrm{n}=5)$, infusão do medicamento parada ou atrasada $(\mathrm{n}=4)$, administração de medicamentos de forma inadequada $(n=4)$ e, outras menos frequentes como a conciliação medicamentosa incorreta, troca de via de administração ou medicamento ou paciente errado/trocado.

Também foi observado, que a maioria das notificações de erros de prescrição foram realizadas pela equipe de farmácia clínica $(\mathrm{n}=11 ; 14,10 \%)$. As unidades que mais notificaram os erros de dispensação foram a UTI $(\mathrm{n}=8 ; 10,26 \%)$, a Clínica Médica $(n=7 ; 8,97 \%)$ e a Pediatria $(n=6 ; 7,69 \%)$. Já as notificações que diziam respeito aos erros de administração de medicamentos foram feitas pela Neurologia e Vascular $(n=9 ; 16,67 \%)$. Com isso, ressaltamos a importância do serviço de farmácia, seja ele hospitalar ou clínico, e da adesão dos profissionais ao fluxo de notificações, assim como àqueles que registraram os erros de dispensação, também como a Neurologia e Vascular que se mostrou bem instruída quanto ao sistema de 
melhorias, notificando na maioria das vezes os seus próprios profissionais.

O perfil assistencial de cada instituição contribui para as diferenças nos tipos de incidentes, sendo importantes na identificação das falhas para que possam ser identificadas e corrigidas conforme a demanda dos setores (Tyll et al., 2019). Um estudo recente evidenciou que as estratégias educacionais, organizacionais e novas tecnologias foram com importante finalidade na minimização dos erros de medicação (Mieiro et al., 2019).

O gerenciamento das ocorrências são fundamentais para a melhoria contínua da qualidade e segurança assistencial prestada pelos serviços de saúde e um sistema eficaz prevê apoio para a notificação dos erros e privacidade da quem notificou a ocorrência (Prates \& Malta, 2017). Apesar de alguns funcionários relacionarem as notificações com penalidades sobre os erros cometidos, este pensamento está sendo desmitificado pois a notificação não apresenta qualquer caráter punitivo e sim, à promoção da melhoria dos processos (ANVISA, 2016).

\section{Considerações Finais}

A fragilidade deste estudo se dá pela omissão dos erros de medicação, tanto para a subnotificação, quanto para o registro dos eventos prevenidos, pois muitas vezes a assistência se torna exaustiva e nem sempre existe tempo hábil para a deliberação de todas as adversidades da rotina e o preenchimento de um formulário de notificação ou uma planilha de registro das atividades realizadas. Mas mesmo com a subnotificação, foram registrados 3343 eventos evitados pela equipe farmacêutica que atua como parte das estratégias para prevenção de eventos adversos no âmbito hospitalar, garantindo maior segurança ao paciente que necessita de cuidados.

Desta forma, o presente estudo contribuiu na identificação dos erros de prescrição e dispensação, auxiliando a gestão do serviço de farmácia na tomada de decisões através do direcionamento de ações com finalidade de promover melhorias na qualidade do serviço de farmácia hospitalar e ainda, retratando importante atuação dos profissionais farmacêuticos e revelando a necessidade da prática contínua na prevenção de eventos adversos.

E com a finalidade de garantir maior segurança na assistência ao paciente, a rotina de notificações de eventos adversos precisa ser realizada de forma efetiva. Para isto, a educação permanente tem função indispensável na melhoria dos processos através da utilização de indicadores de qualidade que são ferramentas importantes para o gerenciamento da assistência e segurança do paciente.

Haja vista os resultados apresentados, sugerimos que novos estudos sejam realizados comparando o que foi avaliado no presente trabalho com o atual modelo, após a implantação da dupla-checagem junto da equipe de enfermagem proporcionada através da estruturação da Farmácia Satélite das UTI's. Além disso, seria interessante realizar estudos antes e após o treinamento das equipes prescritoras, relacionando a eficiência da educação continuada e, também avaliar estratégias específicas de intervenção, como a Switch Therapy.

\section{Agradecimentos}

A todos os membros do Serviço de Farmácia Hospitalar e Clínica que proporcionaram o desenvolvimento deste trabalho por meio da adesão das rotinas implantadas, aos membros do Núcleo da Gestão da Qualidade e Segurança do Paciente por fornecer acesso à todas as notificações referentes aos erros de medicação, à Universidade por financiar o projeto através do pagamento das bolsas do Programa de Residência Farmacêutica e, ao Hospital Universitário por ceder campo de atuação e desenvolvimento de pesquisa. 


\section{Referências}

Anacleto, T. A., Rosa, M. B., Neiva, H. M., \& Martins, M. A. P. (2017). Erro de medicação. In Farmácia Hospitalar Coletânea de práticas e conceitos, 6077. Conselho Federal de Farmácia.

ANVISA, A. N. de V. S. (2016). Implantação do Núcleo de Segurança do Paciente em Serviços de Saúde. In Anvisa. http://portal.anvisa.gov.br/documents/33852/3507912/Caderno+6+-+Implantação+do+Núcleo+de+Segurança+do+Paciente+em+Serviços+de+Saúde/cb237 a40-ffd1-401f-b7fd-7371e495755c

ANVISA, A. N. de V. S. (2019). Suspensão importação do insumo farmacêutico ranitidina. https://www.gov.br/anvisa/pt-br/assuntos/noticiasanvisa/2019/suspensa-importacao-do-insumo-farmaceutico-ranitidina

Araújo, A. P. V., \& Melo, D. O. de. (2018). Substituição da via de administração de medicamentos: atuação do residente farmacêutico e economia de recursos. Jornal de Assistência Farmacêutica e Farmacoeconomia, 14-24. https://doi.org/10.22563/2525-7323.2018.v3.n2.p.14-24

Araujo, E. de O., Viapiana, M., Domingues, E. A. M., Oliveira, G. S. de, \& Polisel, C. G. (2017). Intervenções Farmacêuticas em uma Unidade de Terapia Intensiva em um Hospital Universitário. Revista Brasileira de Farmácia Hospitalar e Serviços de Saúde, 08(3), 25-30. https://doi.org/10.30968/rbfhss.2017.083.005

Araújo, E. S., Modesto, A. C. F., Ferreira, T. X. A. M., Provin, M. P., Lima, D. M., \& Amaral, R. G. (2020). Intervenção farmacêutica no uso racional de omeprazol intravenoso. Einstein (São Paulo), 18(1), 1-6. https://doi.org/10.31744/einstein

Benson, T., \& Grieve, G. (2021). Principles of Health Interoperability. In Springer, Cham. https://doi.org/10.1007/978-3-030-56883-2

Botelho, J. de A., \& Roese, F. M. (2017). Intervenções Realizadas Pelo Farmacêutico Em Uma Unidade De Pronto Atendimento Médico. Revista Brasileira de Farmácia Hospitalar e Serviços de Saúde, 8(1), 34-36. http://www.saude.pr.gov.br/arquivos/File/

Cação, E. de V., Bokehi, J. R., Futuro, D. O., \& De Castilho, S. (2020). O fluxo montante na logística de uma farmácia escola pública: um estudo de caso. RAHIS- Revista de Administração Hospitalar e Inovação Em Saúde, 16(2), 81-93. https://doi.org/10.21450/rahis.v16i2.5773

Chaves, L. A., Chaves, G. C., Vianna, M. N. S., \& Oliveira, M. A. (2019). Desabastecimento de medicamentos na literatura científica da saúde: uma revisão narrativa. Physis: Revista de Saúde Coletiva, 29(1), 1-26. https://doi.org/10.1590/s0103-73312019290107

de Fátima Silva, L., Afonso, T., Veloso e Sousa, C., \& Pellizzaro Dias Afonso, B. (2018). Vulnerabilities and rupture risk in the supply of materials and medicine on the supply chain in the Public Hospital. Revista Gestão Da Produção Operações $e$ Sistemas, 13(2), 21-43. https://doi.org/10.15675/gepros.v13i2.1832

Dias, D., Wiese, L. P. de L., Pereira, E. M., \& Fernandes, F. M. (2019). Avaliação de intervenções clínicas farmacêuticas em uma UTI de um Hospital Público de Santa Catarina. Revista Brasileira de Farmácia Hospitalar e Serviços de Saúde, 9(3), 1-5. https://doi.org/10.30968/rbfhss.2018.093.005

Fernandes, L. L. (2019). A importância do farmacêutico hospitalar juntamente com a equipe multidisciplinar na Unidade de Terapia Intensiva (UTI). Revista Farol, 8(8), 5-21.

Ferreira, A. S., \& Soler, O. (2020). Fortalecimento das estratégias de segurança de pacientes: uma revisão integrativa quantos aos processos de segurança de medicamentos. Research, Society and Development, 9(12). https://doi.org/10.33448/rsd-v9i12.9564

Galvão, A. A., Oliveira, A. M. De, Carvalho, F. B. De, \& Araújo, R. P. C. (2012). Identification and distribution of dispensing errors in a hospital pharmacy: a comparative study in Salvador Bahia. Revista de Ciências Médicas e Biológicas, 11(1), 201-206.

Haino, B. (2013). Gestão da qualidade hospitalar. Saraiva.

ISMP, I. para P. S. no U. de M. (2016). Programa Nacional De Segurança Do Paciente: Indicadores Para Avaliação Da Prescrição, Do Uso E Da Administração De Medicamentos - Parte I. Boletim ISMP Brasil, 5(1), 1-5.

Jesus, A. M. De, Morais, C. R., \& Almeida, O. C. H. (2020). Eventos adversos relacionados à assistência em serviços de saúde: um desafio para segurança do paciente. Ciências Biológicas e Da Saúde, 6(2), 11-22.

Lima, R. F., \& Câmara, T. F. M. (2016). Desafios com o Desabastecimento na Cadeia de Suprimentos em um Hospital Público: Relato de Caso. Revista de Gestão Em Sistemas de Saúde, 5(1), 120-127. https://doi.org/10.5585/rgss.v5i1.218

Maioli, N. A., \& Santos, H. C. B. (2018). Intervenções Farmacêuticas E Sua Importância Na Segurança Do Paciente Hospitalizado. Colloquium Vitae, 10(2), 35-40. https://doi.org/10.5747/cv.2018.v10.n2.v229

Mascarenhas, F. A. de S., Anders, J. C., Gelbcke, F. L., Lanzoni, G. M. de M., \& Ilha, P. (2019). Facilidades e dificuldades dos profissionais da saúde frente ao processo de notificação de eventos adversos. Texto Contexto Enfermagem, 28, 1-15. https://doi.org/10.1590/1980-265x-tce-2018-0040

Mieiro, D. B., Oliveira, É. B. C. de, Fonseca, R. E. P. da, Mininel, V. A., Zem-Mascarenhas, S. H., \& Machado, R. C. (2019). Strategies to minimize medication errors in emergency units: an integrative review. Revista Brasileira de Enfermagem, 72(suppl 1), 307-314. https://doi.org/10.1590/0034-71672017-0658

Miranda, T. M. M., Petriccione, S., Ferracini, F. T., \& Filho, W. M. B. (2012). Intervenções realizadas pelo farmacêutico clínico na unidade de primeiro atendimento. Enstein, Sao Paulo, 10(1), 74-78. https://doi.org/10.1590/S1679-45082012000100015

Mourão, K. Q., \& Oliveira, A. M. de M. (2019). Notificação de eventos: avanços e desafios no contexto da segurança do paciente. Revista Eletrônica Acervo Saúde, 24, 1-6. https://doi.org/10.37885/200700797 
Research, Society and Development, v. 10, n. 3, e18310313280, 2021

(CC BY 4.0) | ISSN 2525-3409 | DOI: http://dx.doi.org/10.33448/rsd-v10i3.13280

MS, M. da S. (2013a). Portaria n 2.095, de 24 de setembro de 2013. Aprova os Protocolos Básicos de Segurança do Paciente. Brasília - DF: Diário Oficial da União.

MS, M. da S. Resolução - RDC 36 de 25 de julho de 2013, (2013).

MS, M. da S., \& ANVISA, A. N. de V. S. (2013). Protocolo de segurança na prescrição, uso e administração de medicamentos. Protocolo coordenado pelo Ministério da Saúde e ANVISA em parceria com FIOCRUZ e FHEMIG. Protocolo Coordenado Pelo Ministério Da Saúde e ANVISA Em Parceria Com FIOCRUZ e FHEMIG, 1-19.

NCCMERP, N. C. C. for M. E. R. P. (1998). Taxonomy of medication errors. https://www.nccmerp.org/taxonomy-medication-errors-now-available.

Pereira, A. S., Shitsuka, D. M., Parreira, F. J., \& Shitsuka, R. (2018). Metodologia da Pesquisa Científica. In Metodologia da Pesquisa Científica (1 ${ }^{\text {a }}$ ed.). https://repositorio.ufsm.br/bitstream/handle/1/15824/Lic_Computacao_Metodologia-Pesquisa-Cientifica.pdf?sequence=1. Acesso em: 28 março 2020.

Perneger, T. V. (2005). The Swiss cheese model of safety incidents: are there holes in the metaphor? BMC Health Services Research, 5(1), 71. https://doi.org/10.1186/1472-6963-5-71

Prates, C. G., \& Malta, M. (2017). Gerenciamento dos incidentes, notificação, investigação e tratamentos. In C. G. Prates \& C. M. Stadñik (Eds.), Segurança do paciente, gestão de riscos e controle de infecções hospitalares (pp. 99-111). Porto Alegre: Moriá.

Reason, J. (2000). Human error: models and management. BMJ, 320(7237), 768-770. https://doi.org/10.1136/bmj.320.7237.768

Resende, A. L. da C., Silva, N. de J., Resende, M. A., Santos, A. A. dos, Souza, G. de, \& Souza, H. C. de. (2020). A importância da notificação de eventos adversos frente à segurança do paciente e à melhoria da qualidade assistencial: uma revisão bibliográfica. Revista Eletrônica Acervo Saúde, (39). https://doi.org/10.25248/reas.e2222.2020

Rezende, L. H. O., Gehrke, F. D. S., Silva, M. A., Carneiro, A. M. F., Abreu, R. M., Monteiro, C. N., \& Takei, K. (2020). Prescrição de medicamentos: uma análise para a implantação da prescrição eletrônica ambulatorial. Revista Eletrônica Acervo Saúde, 12(9), e3638. https://doi.org/10.25248/reas.e3638.2020

Rissato, M. de A. R. (2012). Erros de dispensação de medicamentos em hospital universitário no Paraná. Universidade de São Paulo.

Sirtoli, R., Pinto, E. C. P., Fieira, C., Volkweis, J. G., Penteado, S. T. da S., \& Caldeira, L. de F. (2019). Perfil e análise de pareto dos erros de dispensação de um hospital público. Revista Brasileira de Farmácia Hospitalar e Serviços de Saúde, 9(2), 9-11. https://doi.org/10.30968/rbfhss.2018.092.007

Tyll, M. G., Lima, S. D. C., Carvalho, S. L. S., Peixoto, M. D. S., Cunha, C. L., \& Ferreira, G. R. O. N. (2019). Segurança do paciente: notificação de incidentes em hospitais de referência. Enfermagem Brasil, 18(6), 730-736. https://doi.org/10.33233/eb.v18i6.2834

Vieira, I. R. C. (2019). Segurança do paciente: Diagnóstico de erros de prescrição em um hospital microrregional. Universidade Federal de Outro Preto. 